\title{
IN-PLANE THERMAL CONDUCTIVITY OF PEM FUEL CELL GAS DIFFUSION LAYERS
}

\author{
Ehsan Sadeghi \\ University of Victoria \\ Victoria, BC, Canada
}

\author{
Ned Djilali \\ University of Victoria \\ Victoria, BC, Canada
}

\author{
Majid Bahrami \\ Simon Fraser University \\ Surrey, BC, Canada
}

\section{ABSTRACT}

Heat transfer through the gas diffusion layer $(G D L)$ is a key process in the design and operation of a PEM fuel cell. The analysis of this process requires determination of the effective thermal conductivity. This transport property differs significantly in the through-plane and in-plane directions due to the anisotropic micro-structure of the GDL.

In the present study, a novel test bed that allows the separation of in-plane effective thermal conductivity and thermal contact resistance in GDLs is described. Measurements are performed using Toray carbon paper TGP-H-120 samples for a range of PTFE content at a mean temperature of $65-70^{\circ} \mathrm{C}$. The measurements are complemented by a compact analytical model that achieves good agreement with the experimental data. The in-plane effective thermal conductivity is found to be about 12 times higher than the through-plane conductivity and remains approximately constant, $k \approx 17.5 \mathrm{~W} / \mathrm{mK}$, over a wide range of PTFE content.

\section{NOMENCLATURE}

A cross-sectional area of fluxmeters $\left(\mathrm{m}^{2}\right)$

$A_{\text {in }} \quad$ cross-sectional area of GDL $\left(\mathrm{m}^{2}\right)$

$a \quad$ radius of contact area between fibers (m)

$b \quad$ radius of the area covered by PTFE at contact points (m)

$k \quad$ thermal conductivity (W/mK)

$k_{\text {cell }} \quad$ in-plane effective thermal conductivity of each cell, Fig. $3(\mathrm{~W} / \mathrm{mK})$

$k_{\text {eff }, \text { in }} \quad$ in-plane effective thermal conductivity of GDL $(\mathrm{W} / \mathrm{mK})$

$k_{e f f, i n, 0}$ in-plane effective thermal conductivity of GDL, no PTFE (W/mK) $k_{\text {Parallel }}$ effective thermal conductivity based on the Parallel model (W/mK)

$k_{P T F E} \quad$ thermal conductivity of PTFE (W/mK)

$k_{s} \quad$ thermal conductivity of carbon fiber $(\mathrm{W} / \mathrm{mK})$

$L \quad$ length of GDL sample, distance between sample holders, Figs. 1, 3 (m)

$m$ number of cells

$N \quad$ total number of GDL samples in the experiment

$n \quad$ number of fibers in each cell

$Q \quad$ heat transfer rate (W)

$q \quad$ heat flux $\left(\mathrm{W} / \mathrm{m}^{2}\right)$

$R_{\text {cell }} \quad$ cell thermal resistance (K/W)

$R_{\text {end }} \quad$ thermal resistance at sample ends (K/W)

$R_{f l} \quad$ thermal resistance of fluxmeter (K/W)

$R_{G D L} \quad$ GDL thermal resistance (K/W)

$R_{g r} \quad$ groove thermal resistance (K/W)

$R_{\text {junc }} \quad$ total thermal resistance at each cell interface (K/W)

$R_{\text {layer }}$ summation of GDL and groove thermal resistance $(\mathrm{K} / \mathrm{W})$

$R_{P T F E}$ thermal resistance of PTFE in contact regions of fibers $(\mathrm{K} / \mathrm{W})$

$R_{S H} \quad$ sample holder thermal resistance (K/W)

$R_{\text {tot }} \quad$ total thermal resistance (K/W)

$R_{w} \quad$ thermal resistance of wooden block (K/W)

$r \quad$ fibers' mean radius (m)

$T \quad$ temperature (K)

TCR thermal contact resistance between fibers (K/W)

$t \quad$ sample thickness (m)

$\bar{V} \quad$ average volume of a fiber $\left(\mathrm{m}^{3}\right)$

$V_{s} \quad$ total volume of fibers $\left(\mathrm{m}^{3}\right)$

$V_{\text {tot }} \quad$ total volume of GDL sample $\left(\mathrm{m}^{3}\right)$ 
width (m)

$z \quad$ heat flow direction (m)

\section{Greek symbols}

$\alpha \quad$ radius of contact area between fibers over fiber radius, $a / r$

$\beta \quad$ radius of area covered by PTFE over fiber radius, $b / r$

$\lambda \quad$ weight fraction of PTFE content

$\varepsilon_{0} \quad$ GDL porosity before PTFE treatment

$\theta \quad$ angle between fiber and heat flux directions

$\theta_{m} \quad$ maximum fiber angle

$\phi_{s} \quad$ fiber volume fraction

$\ell \quad$ fiber average length (m)

\begin{tabular}{ll}
\multicolumn{2}{l}{ Subscripts } \\
1 & right side of experiment setup \\
2 & left side of experiment setup \\
low & lower fluxmeter \\
$r$ & radiation \\
up & upper fluxmeter
\end{tabular}

\section{INTRODUCTION}

The temperature distribution in a proton exchange membrane (PEM) fuel cell changes due to electrochemical reaction and associated irreversibilities [1-5]. Accurate knowledge of the temperature distribution and associated heat transfer mechanisms is required to determine various transport phenomena such as water and species transport, reaction kinetics, and rate of phase change. A comprehensive thermal analysis is also required to assess thermal-related phenomena in PEM fuel cell components, specially gas diffusion layer (GDL), which can improve the efficiency, reliability and durability of the system [6-8].

A key thermo-physical property for thermal analysis of fuel cells is the thermal conductivity of the membrane-electrode assembly components, particularly the gas-diffusion layer (GDL) [9, 10]. The fibrous anisotropic micro-structure of a GDL combined with the large differences between the thermal conductivity of the solid (carbon fibers) and fluid (air/water) phases make it challenging to determine the dependence of the effective thermal conductivity on direction.

The majority of the thermal analyses of fuel cells have relied on a simplified model representation that assumes an isotropic thermal conductivity [10-12] that is determined as a combination of the parallel and series models and/or based on the geometric mean of the thermal conductivities of solid and fluid phases [11, 12]. Although the thermal resistance of GDLs for the in-plane direction is higher compared to the throughplane direction, heat transfer to the bipolar plate (BPP) occurs in both directions due to the alternating nature of the land and channel areas [13]. In the few modeling studies that have considered anisotropy, parametric investigations have shown that the prescription of anisotropic properties has a major impact on current density distribution and on the relative importance of limiting transport processes [13-15]. The determination of the in-plane thermal conductivity is therefore an important parameter for thermal analysis and management of PEM fuel cells and stacks.

Theoretical prediction of the in-plane thermal conductivity of GDLs includes the work of Zamel et al. [16] who developed a numerical model to estimate the through-plane and in-plane effective thermal conductivities of a dry untreated carbon paper GDL. They studied the effects of porosity, fiber distribution and compression on the effective thermal conductivity and concluded that the impact of fiber distribution is more pronounced for the through-plane direction than the in-plane direction. The numerical results indicate that porosity is an essential determinant of the effective thermal conductivity of a GDL but not compression. Based on the results, Zamel et al. [16] proposed correlations for the effective thermal conductivity of a dry GDL with no binder or hydrophobic treatment.

GDLs are generally treated with polytetrafluoroethylene (PTFE) to render it hydrophobic and enhance liquid water transport [17]. The effect of PTFE treatment on the thermal transport parameters has been investigated by Khandelwal and Mench [18]. Their measurements for SIGRACET ${ }^{\circledR}$ GDLs showed that for $20 \%$ PTFE content, the through-plane thermal conductivity was reduced by $54 \%$ compared to untreated GDL samples; the thermal contact resistance on the other hand was not significantly affected by the variation of PTFE content. These results are opposite to expected trends from physical considerations; additional PTFE is for instance expected to displace lower conductivity air, and hence results in higher effective thermal conductivity. Karimi et al. [19] investigated the effect of PTFE coating on the through-plane conductivity and thermal contact resistance of SpectraCarb GDLs, and reported that the effective thermal conductivity of PTFE-treated GDLs increased slightly at low compression loads and decreased slightly at higher loads. For low compression loads, they also reported significantly higher thermal contact resistance values for PTFE-treated samples compared to untreated ones. This difference decreased with an increase in applied pressure.

Several experimental approaches have been proposed to measure electrical conductivity of GDLs. Ismail et al. [20] measured the in-plane and through-plane electrical conductivity of SIGRACET ${ }^{\circledR}$ GDLs, and found the in-plane electrical conductivity remains approximately constant with an increase in PTFE content. They also reported that the through-plane electrical contact resistance increases with PTFE content.

The micron scale of the fibers combined with the brittle nature of the GDL structure make it challenging to measure the in-plane thermal conductivity of such random micro-structures, and to the author's knowledge, no experimental data has been reported in the open literature.

This paper presents a combined experimental and theoretical investigation focusing on the determination of the in-plane thermal conductivity of PTFE-coated GDLs. Building on our previous study that dealt with the through-plane conductivity and contact resistance [21], the existing test bed 
was modified and an experimental technique developed that enables the measurement of in-plane thermal conductivity of fibrous porous media and thin films. Toray carbon papers TGP$\mathrm{H}-120$ with different PTFE contents are used in the experiments. The in-plane effective thermal conductivity and contact resistance are deduced from the total thermal resistance measurements by performing a series of experiments with GDL samples of different lengths but similar micro-structures. Furthermore, a compact analytical model is proposed to predict the in-plane thermal conductivity of GDLs as a function of porosity and PTFE content.

\section{EXPERIMENTAL STUDY}

The experimental apparatus and a schematic of the test setup for the in-plane thermal conductivity measurement are shown in Fig. 1. The test chamber consists of a stainless steel base plate and a bell jar enclosing the test column. The test column consists of, from top to bottom: the loading mechanism, the heater block, the upper heat fluxmeter, the sample holders, the sample assembly, the lower fluxmeter, the heat sink (cold plate), and the poly methyl methacrylate (PMMA) layer.

The heater block was made of a flat aluminum block in which a pencil-type electrical heater was installed. The designed cold plate consisted of a hollow copper cylinder, 1.9 $\mathrm{cm}$ high and $15 \mathrm{~cm}$ diameter. Cooling was accomplished using a closed loop water-glycol bath in which the coolant temperature can be set. The cold plate was connected to the chiller unit which adjusts the cold water temperature. A load was applied on the upper wooden block and flux meter to improve the contact between the sample holders and flux meters.

The fluxmeters were made of a standard electrolyte iron material. To measure temperatures along the fluxmeters, six Ttype thermocouples were attached to each fluxmeter at specific locations shown in Fig. 1 (b). The thermal conductivity of the iron fluxmeters was known and used to measure the heat flow rate. The thermal conductivity of the fluxmeters was known and used to measure the heat flow rate. The sample holders were made of aluminum and have two grooves with the width of $1.15 \mathrm{~mm}$. Two T-type thermocouples were attached to each sample holder near the grooves to measure the temperature.

\section{Sample Preparation}

Toray carbon papers TGP-H-120 with the base porosity (untreated GDL porosity) of $78 \%$ were used. The examined samples have a wide range of PTFE content from 5\% to $30 \%$. The thickness of GDL samples was measured using a Mitutoyo digital micrometer with the accuracy of $1 \mu \mathrm{m}$. The measurements were performed 10 times for each sample at different locations and the average values are reported in Tab. 1 . The samples were cut in rectangular shapes with the width of $35 \mathrm{~mm}$ and different lengths.

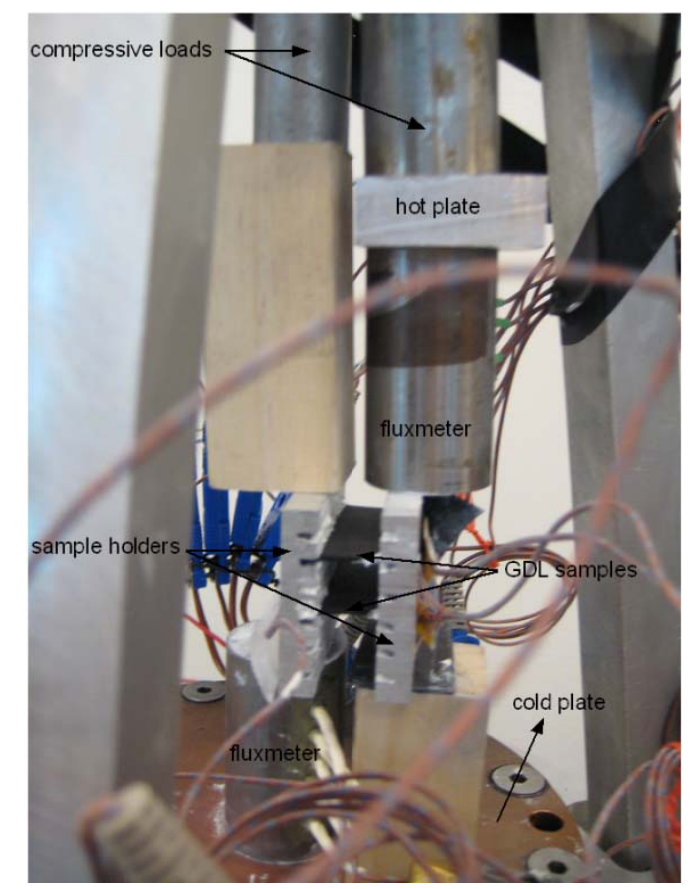

(a)

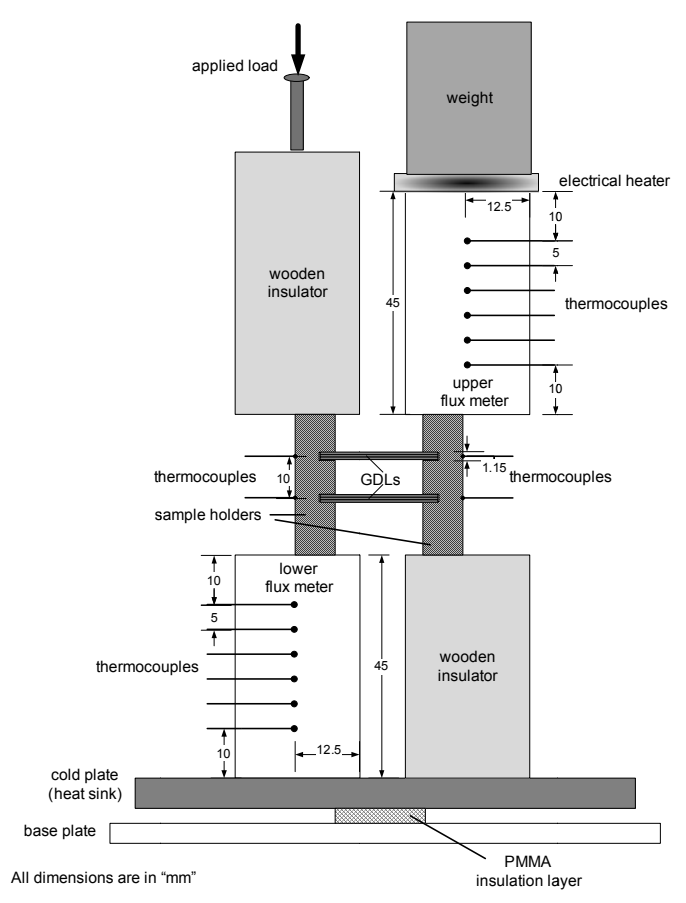

(b)

FIGURE 1. (a) EXPERIMENTAL APPARATUS USED FOR INPLANE THERMAL CONDUCTIVITY MEASUREMENT; (b) SCHEMATIC VIEW OF THE TEST COLUMN 
TABLE 1. THICKNESS VALUES OF EXAMINED TORAY CARBON PAPERS TGP-H-120

\begin{tabular}{ccccc}
\hline PTFE (\%) & 5 & 10 & 20 & 30 \\
\hline $\mathrm{t}(\mathrm{mm})$ & 0.374 & 0.376 & 0.362 & 0.354 \\
\hline
\end{tabular}

\section{Test Procedure}

The experiments were performed under a vacuum to ensure negligible convection heat transfer. Depending on the thickness, a number of similar GDL samples, e.g. three sheets for TGP-H-120, were stacked together and inserted in each groove of the sample holders as shown in Fig. 1. The use of several layers of GDLs mitigated some of the experimental challenges and uncertainties: use of a single GDL layer between the sample holders leads to an excessive temperature drop across the sample holders, which in turn results in insufficient heat flow across the GDL that cannot be measured accurately. This is due to micron size cross-sectional area of the GDL. After investigating and trying different methods, the present sample holders featuring multiple grooves that can hold several GDLs was devised to overcome these challenges.

To reduce the contact resistance between the groove walls and the samples, a thin layer of thermal paste was applied inside each groove. To improve the stability of the sample holders and provide a good contact with the fluxmeters, compressive loads were applied to the upper wooden block and the upper fluxmeter; this is solely to keep the test column together. Thermal paste was also used to reduce the thermal contact resistance at the interfaces between the sample holders and the fluxmeters.

Temperatures were monitored continuously and recorded when steady-state conditions were achieved. This took approximately 7 hours for each experiment. The fairly long equilibration time is due to the restricted cross sectional area through which heat transfer takes place. The temperature gradient between the hot and cold plates results in onedimensional heat conduction from the top to the bottom of the test column. The thermal resistance network corresponding to the experimental set up is shown in Fig. 2. Natural convection heat losses are negligible in the vacuum chamber. Radiation heat losses are investigated; the radiation losses from fluxmeters to wooden blocks are less than $1 \%$ of the total heat flow passing the fluxmeters. Also, small temperature difference between the fibers, in GDLs, as well as relatively low temperature levels, less than $370 \mathrm{~K}$, inside the medium results in a negligible radiation heat transfer in the GDL. Thus, the heat transfer is only due to conduction and can be determined using Fourier's equation.

$$
Q=-k A \frac{d T}{d z}
$$

where, $d T / d z$ is the temperature gradient along the test column, $k$ is the thermal conductivity of the fluxmeters, and
$A$ is the cross-sectional area of fluxmeters. The total thermal resistance between two sample holders, $R_{t o t}$, includes the samples' thermal resistance and resistances at the sample ends (a combination of the thermal contact resistance between the grooves and the samples and other possible resistances caused by the edges of grooves at each end) and can be expressed as:

$$
\begin{aligned}
R_{\text {tot }}=\frac{R_{\text {layer }}}{N}= & \frac{R_{G D L}+R_{\text {gr } 1}+R_{\text {gr } 2}}{N} \\
& =\frac{R_{G D L}}{N}+\frac{R_{\text {end }}}{N}=\frac{\Delta T}{Q}
\end{aligned}
$$

where, $\Delta T$ is the temperature difference between the two sample holders and $N$ is the total number of GDLs layers stacked in the grooves. The reason for using several layers of GDLs is to overcome the experimental challenges and uncertainties. Using a single GDL layer, between the end plates, will lead to tremendous temperature drops across the end plates which leads to very small heat flow across the GDL, which cannot be measured accurately. This is due to low in-plane thermal conductivity and micron size cross-sectional area of the GDL layer (Toray paper). As such, after several trials and investigating different methods, the present end plates featuring multiple grooves that can hold several GDL layers was designed to overcome these challenges.

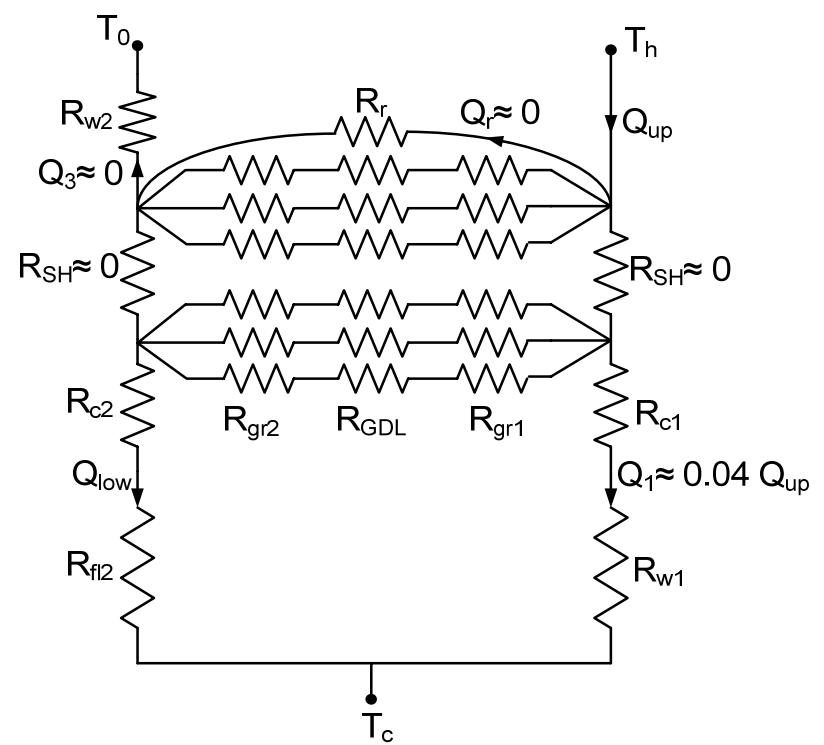

FIGURE 2. RESISTANCE NETWORK OF THE EXPERIMENTAL SET-UP

$R_{G D L}$ and $R_{\text {end }}$ are the thermal resistance of each sample and the total thermal resistance at the end points of each sample, respectively. There is a small difference between the heat flow values measured at the upper and lower fluxmeters due to heat losses to the lower wooden block and also the experiment uncertainties; heat losses to the wooden block is about $4 \%$ of the heat flow passing the upper fluxmeter. 
Therefore, the actual heat flux which passes through the GDL samples is the heat flux measured at the lower flux meter. To have a more accurate result, this heat flow rate was used in the analysis, i.e., Eqn. (2). To find the in-plane thermal conductivity, two sets of experiments were performed with different sample lengths. Under the same experimental conditions, $R_{\text {end }}$ for both experiments was assumed to be equal. Applying Eqn. (2) to both of the measurements and subtracting them, one can find the in-plane effective thermal conductivity.

$$
\begin{aligned}
& k_{e f f, \text { in }}=\frac{L_{1}}{R_{G D L 1} A}=\frac{L_{2}}{R_{G D L 2} A} \\
& k_{e f f, \text { in }}=\frac{N\left(L_{1}-L_{2}\right)}{\left(R_{t o t 1}-R_{t o t 2}\right) A_{\text {in }}}
\end{aligned}
$$

where, $L_{1}$ and $L_{2}$ are the sample lengths, distances between two sample holders in experiment 1 and 2, and $A_{\text {in }}$ is the in-plane cross-section of each sample.

\section{Uncertainty Analysis}

Considering the relationship for evaluating the in-plane effective thermal conductivity, i.e. Eqn. (2) and Eqn. (4), the relevant parameters in the analysis can be expressed as:

$$
k_{e f f, i n}=f(Q, \Delta T, t, W, L)
$$

The main uncertainty in our experiments is due to errors in determining the heat flux through the sample holders which leads to a maximum error of $3.7 \%$. The maximum uncertainties for the thermocouples and the data acquisition readings are $\pm 1^{\circ} \mathrm{C}$ which introduces a maximum error of $1.8 \%$ between two sample holders. Other uncertainties including those associated with the width, thickness, and length measurements are $0.3 \%$, $0.3 \%$, and $0.9 \%$, respectively. The maximum uncertainty for the thermal resistance measurements can be calculated from [22]:

$$
\begin{aligned}
& \frac{\delta k_{e f f, i n}}{k_{e f f, i n}} \\
& =\sqrt{\left(\frac{\delta Q}{Q}\right)^{2}+\left(\frac{\delta \Delta T}{\Delta T}\right)^{2}+\left(\frac{\delta t}{t}\right)^{2}+\left(\frac{\delta W}{W}\right)^{2}+\left(\frac{\delta L}{L}\right)^{2}}
\end{aligned}
$$

For the present study, the maximum uncertainty is estimated to be $\pm 4.2 \%$.

\section{ANALYTICAL STUDY}

The complex micro-structure and associated heat transfer mechanism of fibrous GDLs make it difficult to develop an analytic model for the effective thermal conductivity. To model the in-plane effective thermal conductivity, a random micro- structure divided into $m$ equally-sized cells is considered. Each cell consists of $n$ fibers with an average radius of $r$ and an average length of $\ell$ which are randomly oriented in the $x y$ plane with an angle $\theta$ to the in-plane heat flow direction and stacked vertically in $z$ direction, as shown in Fig. 3. The fiber angle $\theta$ can vary in this representation.

Considering that the primary path for the heat conduction is through the fibers and heat transfer between the fibers in a cell is negligible due to large contact resistances, a parallel equivalent circuit model can be used to determine the thermal conductivity in each cell. The heat conducted through the $i^{\text {th }}$ fiber with an angle $\theta_{i}$ to the heat flux vector $\vec{q}_{i}$ is $q_{i} \cos \theta_{i}$, therefore, each fiber deviates from the Parallel model by $\cos \theta_{i}$. Thus, the effective conductivity for each cell, $k_{\text {cell }}$, can be written as:

$$
k_{\text {cell }}=\frac{\sum_{i=1}^{n} \cos \theta_{i}}{n} k_{\text {Parallel }}=\overline{\cos \theta} \phi_{s} k_{s}
$$

where, $n$ and $\bar{\theta}$ are the number of fibers in a cell and the average angle of fibers with respect to the heat flux direction, respectively. $k_{s}$ and $A_{f}$ is are the fiber thermal conductivity and cross-sectional area, respectively. $\phi_{S}$ is the fiber volume fraction, i.e. $\phi_{s}=1-\varepsilon_{0}$, where $\varepsilon_{0}$ is the GDL porosity before PTFE treatment. We assume that the heat flow is transferred from cell to cell through the junctions. The length of each cell is defined as the average conduction path in a cell, $\ell \overline{\cos \theta}$. Since the fibers are stacked together in a packed microstructure, it is assumed that each fiber of two neighboring cells is in contact with two fibers from the top and bottom and carries heat from them as shown in Fig. 4. The contact between the fibers at a junction is shown in Fig. 5.

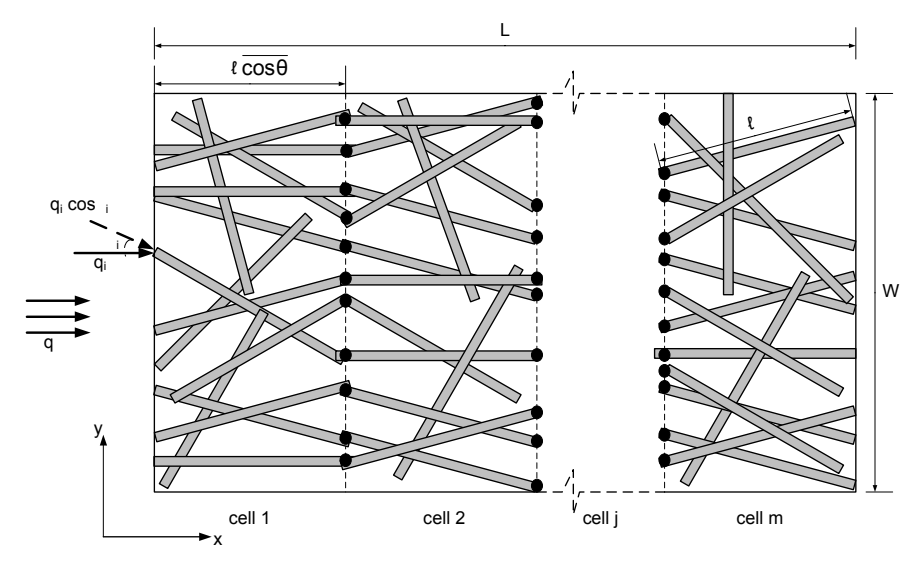

FIGURE 3. CONSTRUCTED MICROSTRUCTURE FOR INPLANE THERMAL CONDUCTIVITY MODELING

To estimate the in-plane effective thermal conductivity of the medium, the total resistance is needed which can be found using the thermal resistance network shown in Fig. 6. The 
number of cells as well as the number of fibers in each cell is required to evaluate the thermal resistances. These values can be found through:

$$
\begin{gathered}
m=\frac{L}{\ell \overline{\cos \theta}} \\
n=\frac{V_{s}}{m \bar{V}}=\frac{\phi_{s} V_{t o t}}{m \bar{V}}=\frac{W t \phi_{s} \overline{\cos \theta}}{\pi r^{2}}
\end{gathered}
$$

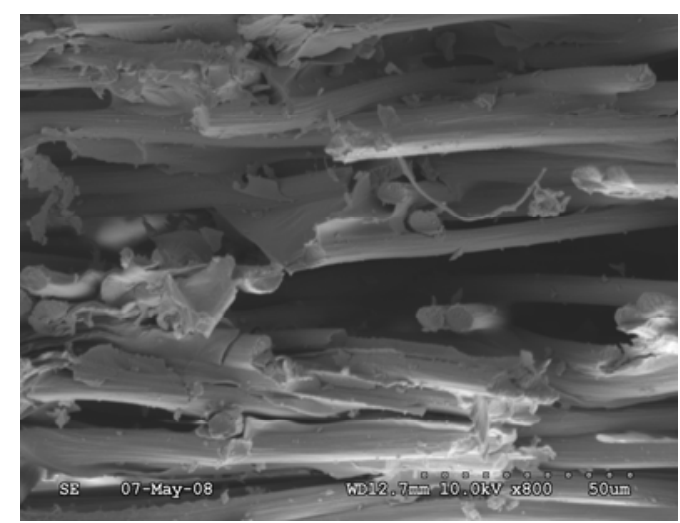

FIGURE 4. IN-PLANE SEM IMAGE OF A TORAY CARBON PAPER, $\times 800$ MAGNIFICATION

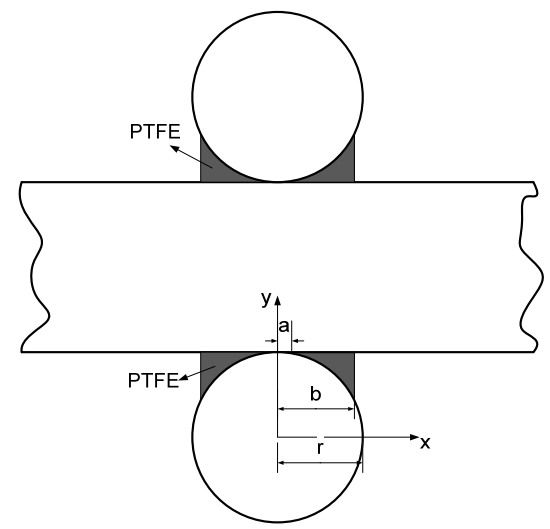

FIGURE 5. CONTACT BETWEEN FIBERS AT A JUNCTION

where, $\bar{V}, W$ and $t$ are the average volume of each fiber, the width and the thickness of the GDL sample. The contact resistance between two fibers can be expressed as the summation of constriction and spreading resistances [23].

$$
T C R=\frac{1}{2 k_{s} a}=\frac{1}{2 k_{s} r \alpha}
$$

where, $a$ is the radius of contact area between fibers and $\alpha$ is $a / r$. A portion of PTFE in GDLs covers the contacting fibers providing an additional path for heat to transfer from one fiber to another. The thermal resistance of this path based on the geometry shown in Fig. 5 can be expressed as:

$$
\begin{aligned}
R_{P T F E}=\left(\int_{a}^{b} \frac{k_{P T F E}(2 \pi x d x)}{2(r-y)}\right)^{-1} & \\
= & \frac{1}{\pi k_{P T F E} r}\left(\int_{\alpha}^{\beta} \frac{x d x}{1-\sqrt{1-x^{2}}}\right)^{-1}
\end{aligned}
$$

where, $\beta=b / r$ and $b$ is the radius of the area covered by PTFE around the contacting fibers, see Fig. 5 . The total thermal resistance at each cell interface $R_{\text {junc }}$ can be expressed as a parallel combination of thermal resistances of $2 n$ contact regions.

$$
R_{\text {junc }}=\frac{\left(T C R^{-1}+R_{P T F E}{ }^{-1}\right)^{-1}}{2 n}
$$
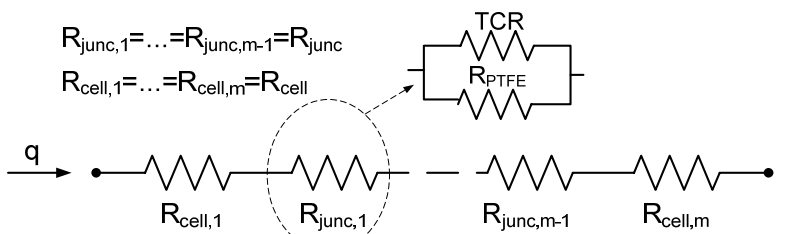

FIGURE 6. THERMAL RESISTANCE NETWORK OF THE INPLANE CONDUCTIVITY MODEL

Referring to Fig. 6, the total resistance can be written as:

$$
R_{\text {tot }}=m R_{\text {cell }}+(m-1) R_{\text {junc }}
$$

where, $R_{\text {cell }}$ is the cell thermal resistance which can be found using Eqn. (7).

$$
R_{\text {cell }}=\frac{\ell}{\phi_{s} k_{s} W t}
$$

Finding the total thermal resistance from Eqn. (13), one can evaluate the effective thermal conductivity using the following relation.

$$
k_{\text {eff,in }}=\frac{L}{R_{\text {tot }} W t}
$$

For the sample with no PTFE coating, Eqn. (15) can be simplified to: 


$$
k_{e f f, i n, 0}=\frac{4 \alpha L \phi_{s} k_{s} \overline{\cos \theta}}{4 \alpha L+\pi(m-1) r} \cong \frac{4 \alpha \ell \phi_{s} k_{s} \overline{\cos \theta}{ }^{2}}{4 \alpha \ell \overline{\cos \theta}+\pi r}
$$

Based on scanning electron microscopy (SEM) images of Toray carbon papers, Fig. 7, the fiber angle with respect to the heat flow direction, $\theta$, can vary arbitrarily between $-\theta_{m}$ and $\theta_{m}$, where $0 \leq \theta_{m} \leq 90$. Considering an arbitrary distribution of fiber angle without preferential direction, $\overline{\cos \theta}$ can be estimated through:

$$
\begin{aligned}
\overline{\cos \theta}=\frac{\sum_{i=1}^{n} \cos \theta_{i}}{n} & =\frac{\sum_{i=1}^{n} \cos \theta_{i} \Delta \theta}{n \Delta \theta} \\
& =\frac{\sum_{i=1}^{n} \cos \theta_{i} \Delta \theta}{2 \theta_{m}}
\end{aligned}
$$

where, $\Delta \theta=\left[\theta_{m}-\left(-\theta_{m}\right)\right] / n$. The number of fibers in a cell is large enough, $n \cong 35000$ to convert the series in Eqn. (17) to an integral.

$$
\overline{\cos \theta}=\frac{\sum_{i=1}^{n} \cos \theta_{i} \Delta \theta}{2 \theta_{m}}=\frac{\int_{-\theta_{m}}^{\theta_{m}} \cos \theta d \theta}{2 \theta_{m}}=\frac{\sin \theta_{m}}{\theta_{m}}
$$

We measured the fiber angle in the SEM image, Fig. 7, and our analysis shows that the majority of fiber angles are between -75 to 75 , i.e. $\theta_{m}=75$. Other specifications of the Toray carbon paper required for the present model are listed in Tab. 2.

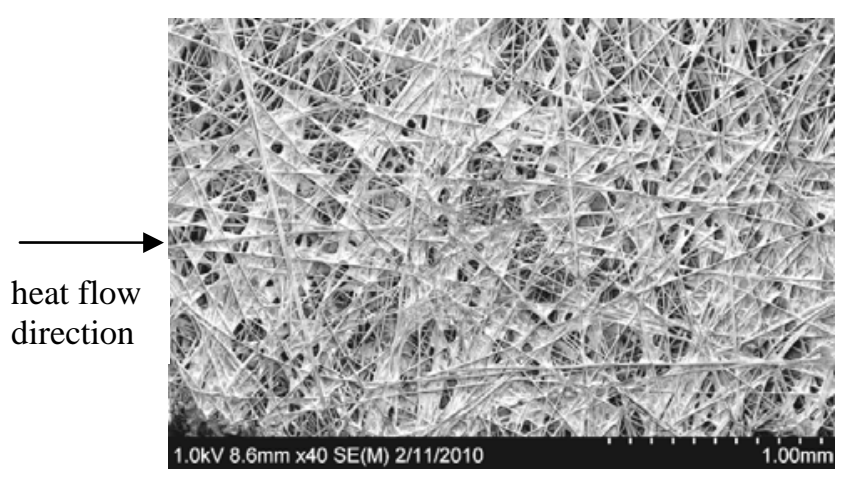

FIGURE 7. SEM IMAGE OF TORAY CARBON PAPER TGP-H120 WITH 5\% PTFE TREATMENT, ×40 MAGNIFICATION

TABLE 2. INPUT DATA FOR THE IN-PLANE THERMAL CONDUCTIVITY MODEL

\begin{tabular}{cccc}
\hline $\mathrm{r}(\mu \mathrm{m})$ & $\ell(\mu \mathrm{m})$ & $\mathrm{k}_{\mathrm{s}}(\mathrm{W} / \mathrm{mK})$ & $\mathrm{k}_{\mathrm{PTFE}}(\mathrm{W} / \mathrm{mK})$ \\
\hline 4.25 & $325[24]$ & $120[25]$ & $0.649[26]$ \\
\hline
\end{tabular}

Toray carbon papers have the highest through-plane thermal conductivity among different available carbon papers with similar porosity due to the contribution of the binder to heat transfer in the carbon paper GDL [16]. The binder fills the gaps between fibers and provides a better contact. The thermal conductivity of the binder can be assumed to be equal to that of the carbon fibers [16]. The actual amount of binders at contact points and as a result the contact area between contacting fibers are unknown. To determine the in-plane thermal conductivity, the value for the radius ratio of contact area to the fiber, $\alpha$, was estimated as 0.1 based on SEM observation shown in Fig. 4.

The PTFE conductivity is very low compared to the thermal conductivity of carbon fibers and its effect on the inplane thermal conductivity is small as shown later. However, to include the effect of PTFE variation in the model, we assume that the radius ratio of PTFE to carbon fiber, $\beta$, is 0.25 for $5 \%$ and 1 for $30 \%$ PTFE content; and remains constant at 1 for higher values of PTFE content. The following relationship is developed for the PTFE content at the contact points of fibers.

$$
\beta=0.25+3(\lambda-0.05)
$$

where, $\lambda$ is the weight fraction of PTFE, $0.05 \leq \lambda \leq 0.3$. This is an approximate relationship, which is proposed based on SEM images of carbon papers with different PTFE contents. However, due to the very low thermal conductivity of PTFE, variants of this distribution do not have a significant impact on the model predictions.

\section{RESULTS AND DISCUSSION}

The measurements were taken for TGP-H-120 samples with different PTFE contents. A summary of experimental results are shown in Tab. 3 . The measurements were performed at an average sample temperature of $65-70^{\circ} \mathrm{C}$. There There is a small difference between the measured values of heat fluxes in the upper and the lower fluxmeters due to heat losses and experimental uncertainties. Due to heat losses to the lower wooden block, the readings from the lower heat flux were used for thermal resistance calculations. As shown in Tab. 3, the inplane thermal conductivity appears to increase slightly with PTFE content as a result of reduced contact resistance between fibers. However, this variation is within the uncertainty band of the present experimental measurement. The thermal conductivity values obtained lie between the values predicted by Zamel et al. [16] and those of the manufacturer [27]. Zamel et al. [16] reported an in-plane thermal conductivity value of $10 \mathrm{~W} / \mathrm{mk}$ based on numerical simulations at a temperature of $68^{\circ} \mathrm{C}$; the value reported in the manufacturer's data sheet [27] is 21 and $23 \mathrm{~W} / \mathrm{mk}$ at the room temperature and $100^{\circ} \mathrm{C}$, respectively.

By increasing PTFE content, the number of PTFE coated fibers in contact with the grooves increases. This results in a higher contact resistance between the GDL samples and the groove walls. This resistance $\mathrm{R}_{\text {end }}$ increases $26 \%$ by increasing PTFE content from $5 \%$ to $30 \%$ as shown in Tab. 3 .

The developed analytical model for the in-plane effective thermal conductivity is compared with the experimental data in Fig. 8. Good agreement is obtained with a maximum deviation of $5 \%$. We note that the model reproduces the slight increase in 
effective thermal conductivity with PTFE content observed in the experimental data.

TABLE 3. SUMMARY OF EXPERIMENTAL RESULTS FOR TORAY CARBON PAPER TGP-H-120 WITH DIFFERENT PTFE CONTENT

\begin{tabular}{|c|c|c|c|c|}
\hline $\begin{array}{c}\text { PTFE } \\
(\%)\end{array}$ & $\begin{array}{c}L \\
(\mathrm{~mm})\end{array}$ & $\begin{array}{c}R_{\text {layer }} \\
(K / W)\end{array}$ & $\begin{array}{c}\mathrm{R}_{\text {end }} \\
(\mathrm{K} / \mathrm{W})\end{array}$ & $\begin{array}{c}\mathrm{k}_{\text {eff,in }} \\
(\mathrm{W} / \mathrm{mK})\end{array}$ \\
\hline 5 & $\begin{array}{l}11.02 \\
14.34\end{array}$ & $\begin{array}{l}63.21 \\
77.79\end{array}$ & 14.27 & 17.39 \\
\hline 10 & $\begin{array}{l}11.20 \\
14.29 \\
\end{array}$ & $\begin{array}{l}65.33 \\
78.88 \\
\end{array}$ & 15.42 & 17.33 \\
\hline 20 & $\begin{array}{l}11.37 \\
14.55 \\
\end{array}$ & $\begin{array}{l}67.13 \\
81.41 \\
\end{array}$ & 17.19 & 17.58 \\
\hline 30 & $\begin{array}{l}11.28 \\
14.67\end{array}$ & $\begin{array}{l}66.95 \\
82.31\end{array}$ & 18.05 & 17.81 \\
\hline
\end{tabular}

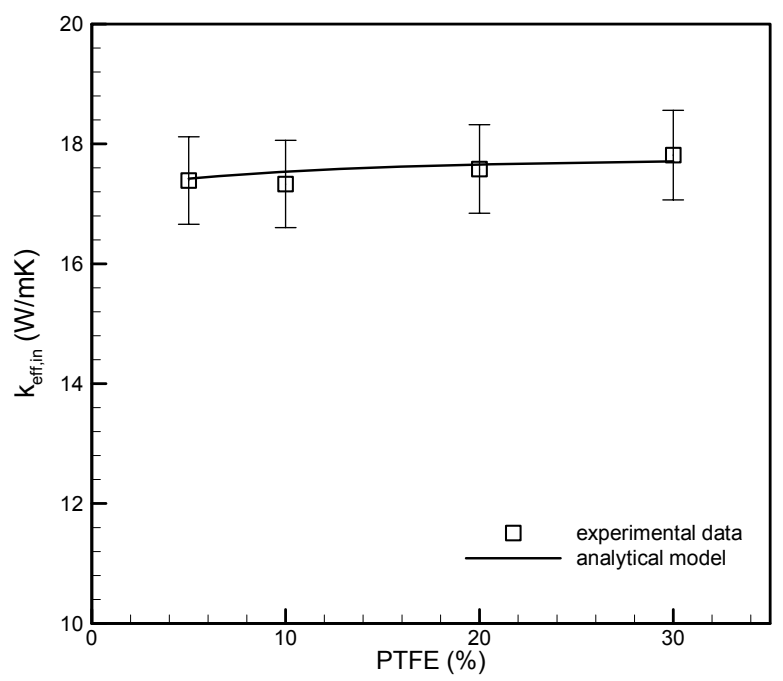

FIGURE 8. COMPARISON OF THE EXPERIMENTAL DATA AND THE ANALYTICAL MODEL FOR THE IN-PLANE THERMAL CONDUCTIVITY OVER A RANGE OF PTFE CONTENT

In practice, due to the electrochemical reaction, overall water transport and phase change, water in both vapor and liquid form is present in a fuel cell [2]. This water may impact the thermal conductivity while passing through the GDL. The thermal conductivity of humidified gasses or water is several orders of magnitude lower than the thermal conductivity of carbon fibers, and considering the relatively parallel paths for the heat transfer through them, the effect of water content on the in-plane thermal conductivity of GDLs is expected to be minimal when the GDL is not significantly flooded. In the through-plane direction, the effect of water is likely much more important as it can provide additional pathways for heat transfer in the contact regions between fibers. Burheim et al. [28] compared the through-plane thermal conductivity of dry and humidified GDLs and showed that the conductivity increases by about $70 \%$ for low contact pressures when water was added to the GDL. This issue needs to be further investigated.

\section{SUMMARY AND CONCLUSIONS}

A new thermal measurement technique was developed to measure the in-plane thermal conductivity of GDLs for various PTFE contents. Toray carbon papers TGP-H-120 with PTFE content of 5 to $30 \%$ were used in the experiments. The experiments were complemented by a compact model for the in-plane thermal conductivity that accounts for heat conduction through randomly oriented fibers, contact area between fibers, and PTFE covered regions. The model predictions are in good agreement with experimental data over a range of PTFE content.

An important finding is that the in-plane effective thermal conductivity remains almost unchanged, $k \approx 17.5 \mathrm{~W} / \mathrm{mK}$, over a wide range of PTFE content; this value is approximately 12 times higher than the through-plane conductivity. However, the thermal contact resistance and the end effects increases with the PTFE content due to increased number of PTFE coated fibers.

In addition to providing for the first time through-plane effective conductivity data, this work clarifies the effect of PTFE content on the effective thermal conductivity and contact resistance of GDLs and provides input data for fuel cell models which require thermal properties of GDLs.

\section{ACKNOWLEDGMENTS}

The authors are grateful for the financial support of the Natural Sciences and Engineering Research Council (NSERC) of Canada, and the Canada Research Chairs Program.

\section{REFERENCES}

[1] Djilali, N., and Lu, D., 2002. "Influence of Heat Transfer on Gas and Water Transport in Fuel Cells". Int. J. Therm. Sci., 41, pp. 29-40.

[2] Berning, T., and Djilali, N., 2003. "A 3D, Multi-Phase, Multicomponent Model of the Cathode and Anode of a PEM Fuel Cell”. J. Electrochem. Soc., 150, pp. A1589A1598.

[3] Maggio, G., Recupero, V., and Mantegazza, C., 1996. "Modelling of Temperature Distribution in a Solid Polymer Electrolyte Fuel Cell Stack ”. J. Power Sources, 62, pp. 167-174.

[4] He, S., Mench, M.M., and Tadigadapa, S., 2006. “Thin Film Temperature Sensor for Real-Time Measurement of Electrolyte Temperature in a Polymer Electrolyte Fuel Cell”. Sensors and Actuators A: Physical, 125, pp. 170-177.

[5] Vie, P.J.S., and Kjelstrup, S., 2004. "Thermal Conductivities from Temperature Profiles in the Polymer 
Electrolyte Fuel Cell”. Electrochim. Acta, 49, pp. 10691077.

[6] Kusoglu, A., Tang, Y., Santare, M.H., Karlsson, A.M., Cleghorn, S., and Johnson, W.B., 2009. "Stress-Strain Behavior of Perfluorosulfonic Acid Membranes at Various Temperatures and Humidities: Experiments and Phenomenological Modeling”. J. Fuel Cell Sci. Technol., 6, pp.011012-011019.

[7] Wilkinson, D.P., Voss, H.H., and Prater, K., 1994. "Water Management and Stack Design for Solid Polymer Fuel Cells”. J. Power Sources, 49, pp. 117-127.

[8] Rama, P., Chen, R., and Andrews, J., 2008. "A Review of Performance Degradation and Failure Modes for Hydrogen-Fuelled Polymer Electrolyte Fuel Cells”. J. Power Energy, 222, pp. 421-441.

[9] Hakenjos, A., Muenter, H., Wittstadt, U., and Hebling, C., 2004. “A PEM Fuel Cell for Combined Measurement of Current and Temperature Distribution, and Flow Field Flooding”. J. Power Sources, 131, pp. 213-216.

[10] Ju, H., Meng, H., and Wang, C.Y., 2005. "A Single-Phase, Non-Isothermal Model for PEM Fuel Cells”. Int. J. Heat Mass Transfer, 48, pp. 1303-1315.

[11] Baschuk, J.J., and Li, X., 2005. "A General Formulation for a Mathematical PEM Fuel Cell Model”. J. Power Sources, 142, pp. 134-153.

[12] Hwang, J.J., 2006. “Thermal-Electrochemical Modeling of a Proton Exchange Membrane Fuel Cell”. J. Electrochem. Soc., 153, pp. A216-A224.

[13] Sui, P.C., and Djilali, N., 2006. “Analysis of Coupled Electron and Mass Transport in the Gas Diffusion Layer of a PEM Fuel Cell”. J. Power Sources, 161, pp. 294-300.

[14] Pharoah, J.G., Karan, K., and Sun, W., 2006. "On Effective Transport Coefficients in PEM Fuel Cell Electrodes: Anisotropy of the Porous Transport Layers”. J. Power Sources, 161, pp.214-224.

[15] Bapat, C.J., and Thynell, S.T., 2008. "Effect of Anisotropic Thermal Conductivity of the GDL and Current Collector Rib Width on Two-Phase Transport in a PEM Fuel Cell”. J. Power Sources, 179, pp.240-251.

[16] Zamel, N., Li, X., Shen, J., Becker, J., and Wiegmann, A., 2010. "Estimating Effective Thermal Conductivity in Carbon Paper Diffusion Media”. Chemical Eng. Sci., 65, pp. 3994-4006.

[17] Park, G.G., Sohn, Y.J., Yang, T.H., Y.G. Yoon, Lee, W.Y., and Kim, C.S., 2004. "Effect of PTFE Contents in the Gas Diffusion Media on the Performance of PEMFC". $J$. Power Sources, 131, pp. 182-187.

[18] Khandelwal, M., and Mench, M.M., 2006. "Direct Measurement of Through-Plane Thermal Conductivity and Contact Resistance in Fuel Cell Materials”. J. Power Sources, 161, pp. 1106-1115.

[19] Karimi, G., Li, X. and Teertstra, P., "Measurement of Through-Plane Effective Thermal Conductivity and Contact Resistance in PEM Fuel Cell Diffusion Media”. Electrochimica Acta, 55, pp. 1619-1625.
[20] Ismail, M.S., Damjanovic, T., Ingham, D.B., Pourkashanian, M., and Westwood, A., 2010. "Effect of Polytetrafluoroethylene-Treatment and Microporous Layer-Coating on the Electrical Conductivity of Gas Diffusion Layers used in Proton Exchange Membrane Fuel Cells” J. Power Sources 195, pp. 2700-2708.

[21] Sadeghi, E., Djilali A., and Bahrami, M., 2011. "Effective Thermal Conductivity and Thermal Contact Resistance of Gas Diffusion Layers in Proton Exchange Membrane Fuel Cells. Part 1: Effect of Compressive Load”. J. Power Sources, 196, pp. 246-254.

[22] Taylor, J.R., 1997. An Introduction to Error Analysis: The Study of Uncertainties in Physical Measurements. $2^{\text {nd }}$ ed., Chap. 3, University Science Books, Sausalito, US.

[23] Carslaw, H.S., and Jaeger, J.C., 1959. Conduction of Heat in Solids. $2^{\text {nd }}$ ed., Oxford Press, London.

[24] Parikh, N., Allen, J., and Yassar, R.S., 2009. "Effect of Deformation on Electrical Properties of Carbon Fibers used in Gas Diffusion Layer of Proton Exchange Membrane Fuel Cells”. J. Power Sources, 193, pp. 766768.

[25] Ramousse, J., Didierjean, S., Lottin, P., and Maillet, D., 2008. "Estimation of the Effective Thermal Conductivity of Carbon Felts Used as PEMFC Gas Diffusion Layers”. Int. J. Therm. Sci., 47, pp.1-6.

[26] Web: http://www.boedeker.com/ptfe_p.htm

[27] Web: http://www.fuelcell.com/techsheets/TORAY-TGPH.pdf

[28] Burheim, O., Vie, P.J.S., Pharoah, J.G., and Kjelstrup, S., 2010. "Ex Situ Measurements of Through-Plane Thermal Conductivities in a Polymer Electrolyte Fuel Cell”. J. Power Sources, 195, pp. 249-256. 